1999-05-02

\title{
The mean spherical approximation for a dipolar Yukawa fluid
}

Douglas Henderson

Dezso Boda

Istvan Szalai

Kwong-Yu Chan

Follow this and additional works at: https://scholarsarchive.byu.edu/facpub

Part of the Biochemistry Commons, and the Chemistry Commons

\section{Original Publication Citation}

Henderson, Douglas, Dezso Boda, Istvjn Szalai, and Kwong Y. Chan."The mean spherical approximation for a dipolar Yukawa fluid." The Journal of Chemical Physics 11 (1999): 7348-7353.

\section{BYU ScholarsArchive Citation}

Henderson, Douglas; Boda, Dezso; Szalai, Istvan; and Chan, Kwong-Yu, "The mean spherical approximation for a dipolar Yukawa fluid" (1999). Faculty Publications. 619.

https://scholarsarchive.byu.edu/facpub/619 


\title{
The mean spherical approximation for a dipolar Yukawa fluid
}

\author{
Douglas Henderson ${ }^{\text {a) }}$ and Dezsö Boda ${ }^{\text {b) }}$ \\ Department of Chemistry and Biochemistry, Brigham Young University, Provo, Utah 84602-5700 \\ István Szalaic) \\ Department of Physical Chemistry, University of Veszprém, H-8201 Veszprém, PO Box 158, Hungary
}

Kwong-Yu Chan ${ }^{\mathrm{d})}$

Department of Chemistry, The University of Hong Kong, Pokfulam Road, Hong Kong

(Received 1 October 1998; accepted 20 January 1999)

\begin{abstract}
The dipolar hard sphere fluid (DHSF) is a useful model of a polar fluid. However, the DHSF lacks a vapor-liquid transition due to the formation of chain-like structures. Such chains are not characteristic of real polar fluids. A more realistic model of a polar fluid is obtained by adding a Lennard-Jones potential to the intermolecular potential. Very similar results are obtained by adding a Yukawa potential, instead of the Lennard-Jones potential. We call this fluid the dipolar Yukawa fluid (DYF). We show that an analytical solution of the mean spherical approximation (MSA) can be obtained for the DYF. Thus, the DYF has many of the attractive features of the DHSF. We find that, within the MSA, the Yukawa potential modifies only the spherically averaged distribution function. Thus, although the thermodynamic properties of the DYF differ from those of the DHSF, the MSA dielectric constant of the DYF is the same as that of the DHSF. This result, and some other predictions, are tested by simulations and are found to be good approximations. (C) 1999 American Institute of Physics. [S0021-9606(99)50815-5]
\end{abstract}

\section{INTRODUCTION}

Wertheim's analytic solution ${ }^{1}$ of the mean spherical approximation (MSA) integral equation for the dipolar hard sphere fluid (DHSF) was the first advance in our understanding of polar fluids since the work of Onsager. ${ }^{2}$ The DHSF fluid has been used in studies of ionic fluids. ${ }^{3}$

Recently, the utility of the DHSF has been called into question. The DHSF has a tendency to form chain-like structures that prevent vapor-liquid coexistence. ${ }^{4}$ Vapor-liquid coexistence will occur if a sufficient amount of dispersion forces is present. Thus, there has been interest recently in the Stockmayer fluid (STMF), where the interaction is the sum of a Lennard-Jones (LJ) potential and a point-dipole interaction. ${ }^{5}$ Unfortunately, the STMF does not lend itself to an analytical solution of any of the usual integral equations used in the theory of fluids. Analytical expressions can be obtained from perturbation theory with suitable simplifications. ${ }^{6,7}$

An alternative procedure would be to use a Yukawa potential to represent the dispersion force. This model, the dipolar Yukawa fluid (DYF), should have many, perhaps all, of the advantages of the STMF. In particular, we show that there is an analytic solution of the MSA for this fluid. Thus, the DYF is at least as useful a model of a polar fluid as is the DHSF.

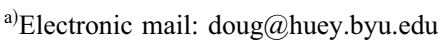

b) Permanent address: Department of Physical Chemistry, University of Veszprém, H-8201 Veszprém, PO Box 158, Hungary; Electronic mail: boda@almos.vein.hu

c)Electronic mail: szalai@almos.vein.hu

${ }^{\mathrm{d})}$ Electronic mail: chemmail@hkucc.hk
}

\section{THEORY}

The pair potential of the DYF is

$$
\begin{aligned}
& u\left(\mathbf{r}_{1}, \mathbf{r}_{2}\right) \\
& \quad=\left\{\begin{array}{l}
\infty, \quad R_{12}<\sigma \\
-\frac{\epsilon_{Y} \sigma}{R_{12}} \exp \left[-\lambda\left(R_{12}-\sigma\right)\right]-\frac{\mu^{2}}{R_{12}^{3}} D(1,2), \quad R_{12}>\sigma,
\end{array}\right.
\end{aligned}
$$

where $\epsilon_{Y}$ and $\lambda$ specify the depth and the range of the dispersion interaction. The subscript $Y$ is used in the energy parameter $\epsilon_{Y}$ to distinguish it from the dielectric constant $\epsilon$. The value $\lambda=1.8 / \sigma$ results in thermodynamic functions of the Yukawa fluid that are similar to those of the LJ fluid. ${ }^{8}$ The parameters $\sigma$ and $\mu$ are the diameter and the dipole moment of a molecule, respectively. Finally,

$$
D(1,2)=3\left(\hat{\boldsymbol{\mu}}_{1} \cdot \hat{\mathbf{R}}_{12}\right)\left(\hat{\boldsymbol{\mu}}_{2} \cdot \hat{\mathbf{R}}_{12}\right)-\Delta(1,2),
$$

where

$$
\Delta(1,2)=\hat{\boldsymbol{\mu}}_{1} \cdot \hat{\boldsymbol{\mu}}_{2},
$$

$\boldsymbol{\mu}_{i}$ is the dipole moment, with direction, of molecule $i$,

$$
\mathbf{R}_{12}=\mathbf{r}_{1}-\mathbf{r}_{2},
$$

and

$$
R_{12}=\left|\mathbf{R}_{12}\right| \text {. }
$$

The caret over the vector indicates that the vector is a unit vector, i.e., $\hat{\mathbf{R}}_{12}=\mathbf{R}_{12} / R_{12}$ and $\hat{\boldsymbol{\mu}}_{i}=\boldsymbol{\mu}_{i} / \mu$. The three functions, $1, \Delta(1,2)$, and $D(1,2)$ are orthogonal functions. 
The MSA is based upon the combination of the Ornstein-Zernike $(\mathrm{OZ})$ relation

$$
h(1,2)=c(1,2)+\rho \int h(1,3) c(2,3) d \mathbf{r}_{3},
$$

where $g(1,2)=h(1,2)+1$ is the pair distribution function (PDF), and $c(1,2)$ is the direct correlation function (DCF), with

$$
\begin{aligned}
& h(1,2)=-1, \quad R_{12}<\sigma, \\
& c(1,2)=-\beta u(1,2), \quad R_{12}>\sigma .
\end{aligned}
$$

The function $h(1,2)$ is usually called the total correlation function (TCF). The symbol $\beta=1 / k T$, where $k$ is the Boltzmann constant and $T$ is the temperature. The symbol $\rho$ $=N / V$, where $N$ is the number of molecules in the system and $V$ is the volume. For simplicity, we use the notation $g(1,2)=g\left(\mathbf{r}_{1}, \mathbf{r}_{2}\right)$. The OZ relation is merely a definition of the DCF. The first part of Eq. (7) is a statement of the fact that the molecules cannot overlap because the pair potential is infinite inside the molecular core. The second part of Eq. (7) is the approximation.

We follow the procedure of Wertheim in obtaining the solution of the MSA for the DYF. Because the MSA is a linear response type theory, the three functions $1, \Delta(1,2)$, and $D(1,2)$ are a complete orthogonal basis set. Other spherical harmonics cannot appear. Thus,

$$
g(1,2)=g_{S}\left(R_{12}\right)+h_{\Delta}\left(R_{12}\right) \Delta(1,2)+h_{D}\left(R_{12}\right) D(1,2),
$$

with a similar expansion for $c(1,2)$. The projections, $g_{S}\left(R_{12}\right), h_{\Delta}\left(R_{12}\right)$, and $h_{D}\left(R_{12}\right)$, are given by

$$
\begin{aligned}
& g_{S}\left(R_{12}\right)=\int g(1,2) d \Omega_{1} d \Omega_{2}, \\
& h_{\Delta}\left(R_{12}\right)=3 \int \Delta(1,2) g(1,2) d \Omega_{1} d \Omega_{2},
\end{aligned}
$$

and

$$
h_{D}\left(R_{12}\right)=\frac{3}{2} \int D(1,2) g(1,2) d \Omega_{1} d \Omega_{2},
$$

where $\Omega_{i}$ is the angle defining the orientation of molecule $i$.

Following Wertheim, we use the Fourier transform,

$$
\tilde{f}(k)=\int f(R) \exp [i \mathbf{k} \cdot \mathbf{R}] d \mathbf{R},
$$

to obtain the solution of our equation. The Fourier transform of $g_{S}\left(R_{12}\right)$ and $h_{\Delta}\left(R_{12}\right)$ is obtained easily using

$$
\begin{aligned}
\tilde{f}(k) & =\frac{4 \pi}{k} \int_{0}^{\infty} R f(R) \sin (k R) d R \\
& =4 \pi \int_{0}^{\infty} R^{2} f(R) j_{0}(k R) d R .
\end{aligned}
$$

The Fourier transform of $h_{D}(1,2) D(1,2)$ is more complex since $D(1,2)$ is a function of $\mathbf{r}_{1}$ and $\mathbf{r}_{2}$. However, using Eq. (12),

$$
F T\left[h_{D}\left(R_{12}\right) D(1,2)\right]=\overline{h_{D}}(k) D_{k}(1,2),
$$

where

$$
D_{k}(1,2)=3\left(\hat{\boldsymbol{\mu}}_{1} \cdot \hat{\mathbf{k}}\right)\left(\hat{\boldsymbol{\mu}}_{2} \cdot \hat{\mathbf{k}}\right)-\left(\hat{\boldsymbol{\mu}}_{1} \cdot \hat{\boldsymbol{\mu}}_{2}\right),
$$

and $\bar{h}(k)$ is given by the Hankel transform

$$
\overline{h_{D}}(k)=-4 \pi \int_{0}^{\infty} R^{2} j_{2}(k R) h_{D}(R) d R .
$$

The functions $j_{0}(k R)$ and $j_{2}(k R)$ in Eqs. (13) and (16) are the spherical Bessel functions.

It is convenient to rewrite Eq. (14) as

$$
F T\left[h_{D}\left(R_{12}\right) D(1,2)\right]=\tilde{H}_{D}(k) D_{k}(1,2),
$$

where $\widetilde{H}_{D}(k)$ is the Fourier transform of $H_{D}\left(R_{12}\right)$, which is related to $h_{D}\left(R_{12}\right)$ by

$$
H_{D}(R)=h_{D}(R)-3 \int_{R}^{\infty} \frac{h_{D}\left(R^{\prime}\right)}{R^{\prime}} d R^{\prime} .
$$

Because of the orthogonality of the basis functions, the transformed OZ relation decomposes into the equations

$$
\begin{aligned}
& \tilde{h}_{S}(k)=\tilde{c}_{S}(k)+\rho \tilde{h}_{S}(k) \tilde{c}_{S}(k), \\
& \tilde{h}_{\Delta}(k)=\tilde{c}_{S}(k)+\frac{1}{3} \rho\left[\tilde{h}_{\Delta}(k) \tilde{c}_{\Delta}(k)+2 \tilde{H}_{D}(k) \widetilde{C}_{D}(k)\right],
\end{aligned}
$$

and

$$
\begin{aligned}
\tilde{H}_{D}(k)= & \widetilde{C}_{D}(k)+\frac{1}{3} \rho\left[\widetilde{h}_{\Delta}(k) \widetilde{C}_{D}(k)+\tilde{H}_{D}(k) \tilde{c}_{\Delta}(k)\right. \\
& \left.+\widetilde{h}_{D}(k) \widetilde{C}_{D}(k)\right] .
\end{aligned}
$$

The function $C_{D}(R)$ and its transform $\widetilde{C}_{D}(k)$ are defined analogously to $H_{D}(R)$ and $\widetilde{H}_{D}(k)$. Note that Eq. (19) is decoupled from Eqs. (20) and (21). The functions $h_{S}(R)$ and $c_{S}(R)$ satisfy

$$
\begin{aligned}
& h_{S}(R)=-1, \quad R<\sigma, \\
& c_{S}(R)=\frac{\beta \epsilon_{Y} \sigma}{R} \exp [-\lambda(R-\sigma)], \quad R>\sigma .
\end{aligned}
$$

Thus, $h_{S}(R)$ and $c_{S}(R)$ are precisely the TCF and DCF that results from the solution of the MSA for the Yukawa fluid, i.e., the fluid defined by Eq. (1), with $\mu=0$. The DCF and thermodynamics of this fluid have been given by Waisman. ${ }^{9}$ Waisman's formulas have been simplified. ${ }^{10}$

The functions $h_{\Delta}(R), H_{D}(R), c_{\Delta}(R)$, and $C_{D}(R)$ satisfy

$$
\begin{array}{ll}
h_{\Delta}(R)=0, & R<\sigma, \\
c_{\Delta}(R)=0, & R>\sigma,
\end{array}
$$

and

$$
\begin{aligned}
& H_{D}(R)=-3 K, \quad R<\sigma, \\
& C_{D}(R)=0, \quad R>\sigma,
\end{aligned}
$$

where

$$
K=\int_{\sigma}^{\infty} \frac{h_{D}(R) d R}{R},
$$

is a constant that is to be determined. 
Wertheim has shown that the solution of Eqs. (20), (21), (23), and (24) is

$$
h_{\Delta}(R)=2 K\left[h_{\mathrm{HS}}^{\mathrm{PY}}(R ; 2 K \rho)-h_{\mathrm{HS}}^{\mathrm{PY}}(R ;-K \rho)\right],
$$

and

$$
H_{D}(R)=2 K\left[h_{\mathrm{HS}}^{\mathrm{PY}}(R ; 2 K \rho)+\frac{1}{2} h_{\mathrm{HS}}^{\mathrm{PY}}(R ;-K \rho)\right],
$$

where $h_{\mathrm{HS}}^{\mathrm{PY}}(R ; \xi)$ is the solution of the Percus-Yevick (PY) equation for hard spheres (HS) for a given distance, $R$, and density, $\xi$, i.e., the fluid given by Eq. (1) with both $\epsilon_{Y}=0$ and $\mu=0$. The equations for $c_{\Delta}(R)$ and $C_{D}(R)$ are analogous to Eqs. (26) and (27). theim:

The parameter $K$ is given by the formula due to Wer-

$$
\frac{(1+4 K \eta)^{2}}{(1-2 K \eta)^{4}}-\frac{(1-2 K \eta)^{2}}{(1+K \eta)^{4}}=3 y,
$$

where $\eta=\pi \rho \sigma^{3} / 6$ and $y=4 \pi \rho \beta \mu^{2} / 9$. Note that $0<K \eta$ $<1 / 2$.

The dielectric constant is given by Wertheim's formula:

$$
\epsilon=\frac{(1+4 K \eta)^{2}(1+K \eta)^{4}}{(1-2 K \eta)^{6}} .
$$

Because the equations for $g_{S}(R)$ and $c_{S}(R)$, and those for $h_{\Delta}(R), c_{\Delta}(R), H_{D}(R)$, and $C_{D}(R)$ decouple in the MSA, the thermodynamics will also be additive. This cannot be exactly true because simulation studies for the DHSF have shown that $g_{S}(R)$ increases slightly near contact as the dipole moment is turned on. However, it is to be hoped that it will be true, within a reasonable approximation.

The energy is given by

$$
\frac{E-E_{0}}{N k T}=-3 K y,
$$

where $E_{0} / N k T$ is the energy of the YF. Assuming that the energy is the best route to the free energy and pressure, we have

$$
\frac{A-A_{0}}{N k T}=-K^{2} \eta\left[8 \frac{(1+K \eta)^{2}}{(1-2 K \eta)^{4}}+\frac{(2-K \eta)^{2}}{(1+K \eta)^{4}}\right],
$$

and

$$
\frac{p-p_{0}}{\rho k T}=K^{2} \eta\left[8 \frac{(1+K \eta)^{2}}{(1-2 K \eta)^{4}}+\frac{(2-K \eta)^{2}}{(1+K \eta)^{4}}\right]-3 K y,
$$

where $E_{0}, A_{0}$, and $p_{0}$ are the values of these functions for the YF. These can be calculated implicitly from the formulas of Waisman, with the simplifications of Høye and Stell and Ginoza or explicitly from the approximate formulas of Henderson et al. ${ }^{10}$

Before examining the properties of the DYF, we first investigate the independence of $g_{S}(R), h_{\Delta}(R)$, and $h_{D}(R)$ by simulations. Firstly, we start with a YF and examine the degree to which $g_{S}(R)$ changes as $\mu$ is increased at constant $\epsilon_{Y}$. Secondly, we start with the DHSF and examine the degree to which $h_{\Delta}(R), h_{D}(R)$, and $\epsilon$ change as $\epsilon_{Y}$ is increased at constant $\mu$ and constant temperature. Thirdly, we report some results for vapor-liquid equilibria in the DYF. For the parameters chosen, there is no evidence of chain-like structures.

\section{SIMULATIONS}

The bulk simulations were performed in the canonical $(N V T)$ ensemble using the usual Boltzmann sampling and periodic boundary conditions. A spherical cutoff of half the cell length was applied, and long range corrections (LRC) were taken into account. The LRC of the dipole-dipole interaction was calculated on the basis of the reaction field (RF) method ${ }^{11}$ using the conducting boundary condition $\left(\epsilon_{\mathrm{RF}} \rightarrow \infty\right)$, while the LRC of the Yukawa-tail was obtained from

$$
U_{Y}^{\mathrm{LRC}}=-2 \pi N \rho \epsilon_{Y} \sigma\left(R_{c} \lambda^{-1}+\lambda^{-2}\right) \exp \left[-\lambda\left(R_{c}-\sigma\right)\right],
$$

where $R_{c}$ is the cutoff radius.

The dielectric constant of the fluid can be obtained from the equation

$$
\frac{(\epsilon-1)\left(2 \epsilon_{\mathrm{RF}}+1\right)}{\epsilon+2 \epsilon_{\mathrm{RF}}}=3 y g_{\mathrm{K}},
$$

where $g_{\mathrm{K}}$ is the Kirkwood $g$-factor, and it is related to the fluctuation of the total dipole moment in the simulation cell in following manner:

$$
g_{K}=\frac{\left\langle M^{2}\right\rangle-\langle M\rangle^{2}}{N \mu^{2}}=\frac{\left\langle M^{2}\right\rangle}{N \mu^{2}} .
$$

The latter equality is due to the fact that in zero field $\langle M\rangle$ $=0$.

Simulations were performed at reduced density $\rho \sigma^{3}$ $=0.8$ for $\lambda=1.8 / \sigma$ for various values of $\beta \epsilon_{Y}$ and $\beta \mu^{2} / \sigma^{3}$. Some results for the contact value of the radial distribution function, $g_{S}\left(\sigma^{+}\right)$, the energy, the pressure, and the dielectric constant can be seen in Table I. The results obtained for YF agree well with previous $\mathrm{MC}^{8}$ and molecular dynamics ${ }^{12}$ data. Further, the results obtained for the DHS show good agreement with the simulation results of Levesque et al. ${ }^{13,14}$ and Lomba et al. ${ }^{15}$ The simulation values for hard spheres (HSF) are those of Barker and Henderson. ${ }^{16}$

From Table I it is evident that turning on the Yukawatail has a strong effect on the energy of the fluid. The dielectric constant, on the contrary, is relatively insensitive to changes in the value of $\beta \epsilon_{Y}$. This is exactly what the MSA predicts.

Also the distribution functions $g_{S}(R), h_{\Delta}(R)$, and $h_{D}(R)$ behave according to the predictions of the MSA. Figure 1 shows $g_{S}(R)$ of the DYF for various dipole moments, while in Figs. 2 and 3 the projections $h_{D}(R)$ and $h_{\Delta}(R)$ can be seen for various values of $\beta \epsilon_{Y}$. It is seen that $g_{S}(R)$ is relatively insensitive to $\beta \mu^{2} / \sigma^{3}$. Of course, as is seen in Table I, some change occurs at contact. The functions $h_{D}(R)$ and $h_{\Delta}(R)$ do not depend on $\beta \epsilon_{Y}$, at least at the studied parameters of the DYF.

In Figs. 4 and 5 we report MSA and simulation values for vapor-liquid coexistence in the DYF for $\beta \mu^{2} / \sigma^{3}=0$ and 1. The simulation results were obtained using the extended 
TABLE I. Comparison of simulation (MC) and DYF MSA results. $\rho \sigma^{3}=0.8, \lambda=1.8 / \sigma$ and $N=256$.

\begin{tabular}{|c|c|c|c|c|c|c|c|c|c|c|}
\hline \multirow[t]{2}{*}{ System } & \multirow[t]{2}{*}{$\beta \epsilon_{Y}$} & \multirow[t]{2}{*}{$\beta \mu^{2} / \sigma^{3}$} & \multicolumn{2}{|c|}{$g\left(\sigma^{+}\right)$} & \multicolumn{2}{|c|}{ U/NkT } & \multicolumn{2}{|c|}{$p \sigma^{3} / k T$} & \multicolumn{2}{|c|}{$\epsilon$} \\
\hline & & & $\mathrm{MC}$ & MSA & $\mathrm{MC}$ & MSA & $\mathrm{MC}$ & MSA & $\mathrm{MC}$ & MSA \\
\hline HSF & 0 & 0 & 3.97 & 3.58 & 0 & 0 & 6.12 & 6.199 & 1 & 1 \\
\hline YF & 1 & 0 & 4.43 & 3.68 & -5.64 & -5.616 & 0.96 & 0.909 & 1 & 1 \\
\hline DHSF & 0 & 1 & 4.28 & 3.58 & -1.03 & -0.688 & 5.71 & 5.970 & 8.8 & 7.799 \\
\hline DYF & 0.5 & 1 & 4.45 & 3.63 & -3.85 & -3.492 & 3.05 & 3.316 & 8.0 & 7.799 \\
\hline DYF & 1 & 1 & 4.66 & 3.68 & -6.68 & -6.304 & 0.43 & 0.680 & 8.6 & 7.799 \\
\hline DHSF & 0 & 2 & 4.74 & 3.58 & -2.85 & -1.992 & 4.87 & 5.612 & 29.5 & 20.001 \\
\hline DYF & 0.5 & 2 & 4.96 & 3.63 & -5.62 & -4.796 & 2.31 & 2.959 & 31.3 & 20.001 \\
\hline DYF & 1 & 2 & 5.21 & 3.68 & -8.47 & -7.608 & -0.27 & 0.323 & 32.7 & 20.001 \\
\hline
\end{tabular}

NpT plus test particle $(\mathrm{NpT}+\mathrm{TP})$ method, described in details in our previous work. ${ }^{17,18}$ We only give an outline here. Prescribing the point $\left(\beta_{0}, p_{0}\right)$ in the $\beta, p$ parameter plane, the reduced chemical potential $(\beta g)$ can be expanded into a two dimensional Taylor series about the point $\left(\beta_{0}, p_{0}\right)$ up to third order:

$$
\begin{aligned}
\beta g(\beta, p)= & \beta_{0} g\left(\beta_{0}, p_{0}\right)+\sum_{n=1}^{3} \frac{1}{n !}\left[\left(\beta-\beta_{0}\right) \frac{\partial}{\partial \beta}+(p\right. \\
& \left.\left.-p_{0}\right) \frac{\partial}{\partial p}\right]^{n} \beta g(\beta, p) .
\end{aligned}
$$

The coefficients of the series can be derived from simple thermodynamic relations on the basis of the derivatives of the enthalpy and volume of the system with respect to $\beta$ and $p$ and can be calculated from fluctuation formulas by performing an $\mathrm{NpT}+\mathrm{TP} \mathrm{MC}$ simulation at the $\left(\beta_{0}, p_{0}\right)$ point. All these derivatives and fluctuation formulas have been given. ${ }^{17}$ Performing this procedure for a gas and a liquid system phase point, and rewriting the third order Taylor series of $\beta g$ for these points, the vapor pressure curve as well as other equilibrium data can be obtained from the intersection of these curves in the appropriate temperature range to within a desired accuracy. The NpT ensemble MC simulations involving 256 particles and about 1 million cycles were performed. The chemical potential was calculated by $\mathrm{Wi}$ -

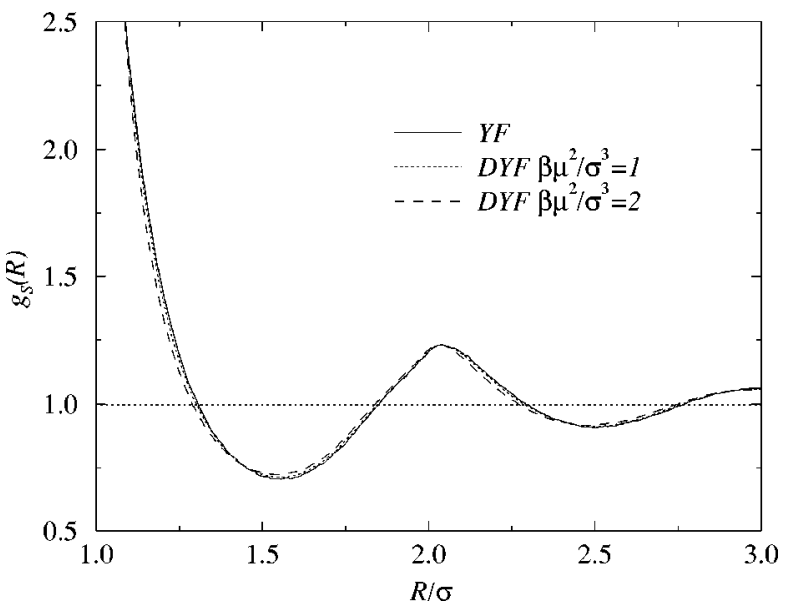

FIG. 1. $g_{S}(R)$ of the YF $(\mu=0)$ and DYF at $\beta \epsilon_{Y}=1$. dom's test particle method (256 test particle insertions for both the liquid and gas phases were used in a single cycle). The agreement of the MSA and simulation coexistence curves is quite good. We find that our results for Yukawa fluid are essentially the same as the results from the literature, ${ }^{19}$ which proves that the program is reliable. We have examined the question of whether chain-like structures of the dipoles are formed. None were found. Of course, if $\mu$ is increased to an arbitrarily large value, such chains can occur. The question of what value of $\beta \mu^{2} / \sigma^{3}$ is necessary for such structures to occur will be considered in later work.

Some discussion of the system size is needed. Levesque et al. ${ }^{16}$ found that $h_{\Delta}(r)$ of DHSF is very sensitive to the number of particles used in the MC simulation. This phenomenon is less apparent in our case. As can be seen in Fig. $6, h_{\Delta}(R)$ is not very sensitive to the number of particles, except at long range. The functions $h_{D}(R)$ and $h_{\Delta}(R)$ should approach zero at large $R$. Larger values of $N$ satisfy this condition better. This may be the consequence of the conducting boundary condition applied in the framework of the reaction field construction. Levesque et al. used a spherical cutoff, a minimum image, and in some cases the RF method with a low finite value of $\epsilon_{\mathrm{RF}}$. Nevertheless, in a later publication, ${ }^{14}$ they used a higher $\epsilon_{\mathrm{RF}}$, and found that $h_{\Delta}(R)$ lies closer to that obtained from integral equation theory for an infinite system.

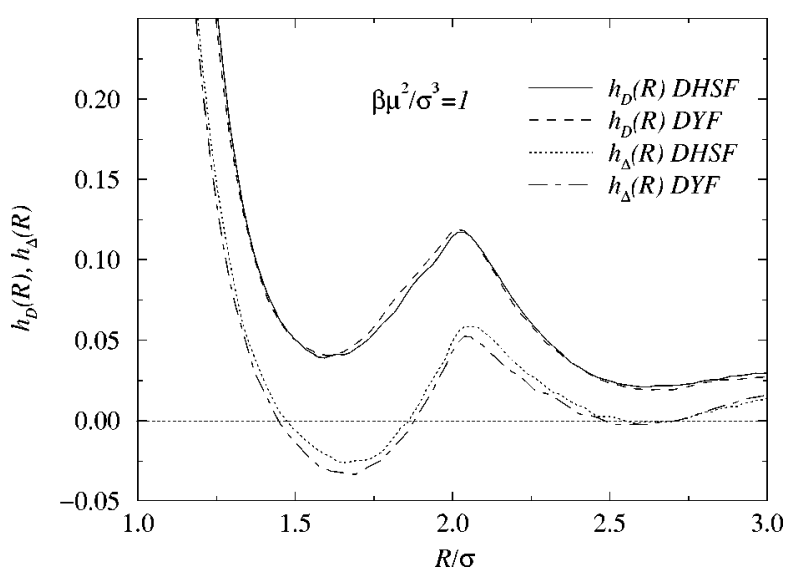

FIG. 2. $h_{D}(R)$ and $h_{\Delta}(R)$ of the DHSF $\left(\beta \epsilon_{Y}=0\right)$ and DYF $\left(\beta \epsilon_{Y}=1\right)$ at $\beta \mu^{2} / \sigma^{3}=1$. 


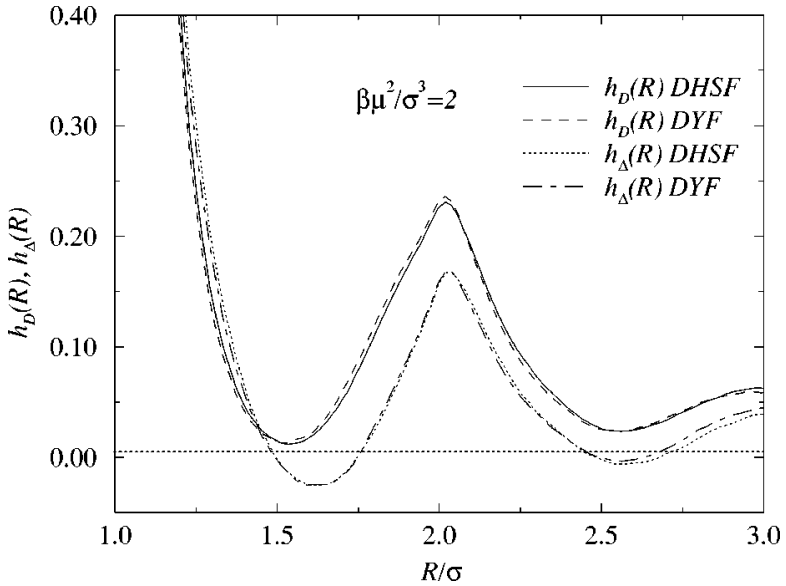

FIG. 3. $h_{D}(R)$ and $h_{\Delta}(R)$ of the DHSF $\left(\beta \epsilon_{Y}=0\right)$ and DYF $\left(\beta \epsilon_{Y}=1\right)$ at $\beta \mu^{2} / \sigma^{3}=2$.

In general, the value of $\epsilon_{\mathrm{RF}}$ influences only the Kirkwood-factor and $h_{\Delta}(R)$, while the dielectric constant is rather independent of the applied boundary condition (i.e., the value of $\epsilon_{\mathrm{RF}}$ ). Note that the system size dependence of $h_{\Delta}(R)$ is stronger at lower values of $\epsilon_{\mathrm{RF}}$.

Because of the absence of a strong system size dependence of $h_{\Delta}(R)$ found in the conducting boundary condition $\left(\epsilon_{\mathrm{RF}} \rightarrow \infty\right)$ applied, we used 256 particles in the simulations tabulated in Table I.

\section{SUMMARY AND CONCLUSION}

In this paper, we have obtained the solution of the MSA for the DYF. The main feature of the solution is that the radial distribution function $g_{S}(R)$ and the angular correlation functions $h_{D}(R)$ and $h_{\Delta}(R)$ are decoupled. The function $g_{S}(R)$ depends on the strength, $\epsilon_{Y}$ of the Yukawa potential and not on the dipole moment, $\mu$, whereas $h_{D}(R)$ and $h_{\Delta}(R)$ depend on $\mu$ and not on $\epsilon_{Y}$.

The MSA values of $g_{S}(R), h_{D}(R)$, and $h_{\Delta}(R)$ are compared with our simulations for the DYF. Although the MSA thermodynamic functions are quite reasonable for the DHSF, these distribution functions are known to be in error for the

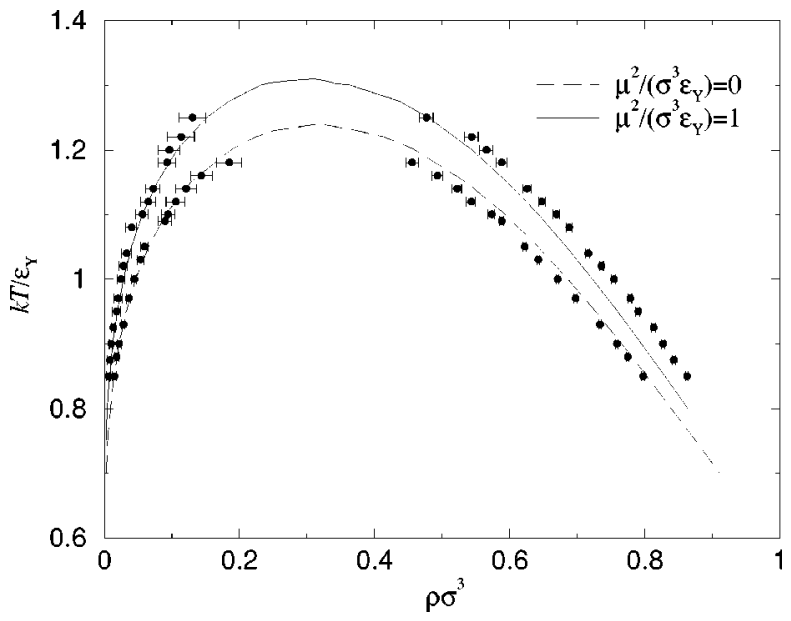

FIG. 4. Vapor-liquid coexistence curves for the DYF for $\beta \mu^{2} / \sigma^{3}=0$ and 1 obtained from simulation (points) and the MSA (curves).

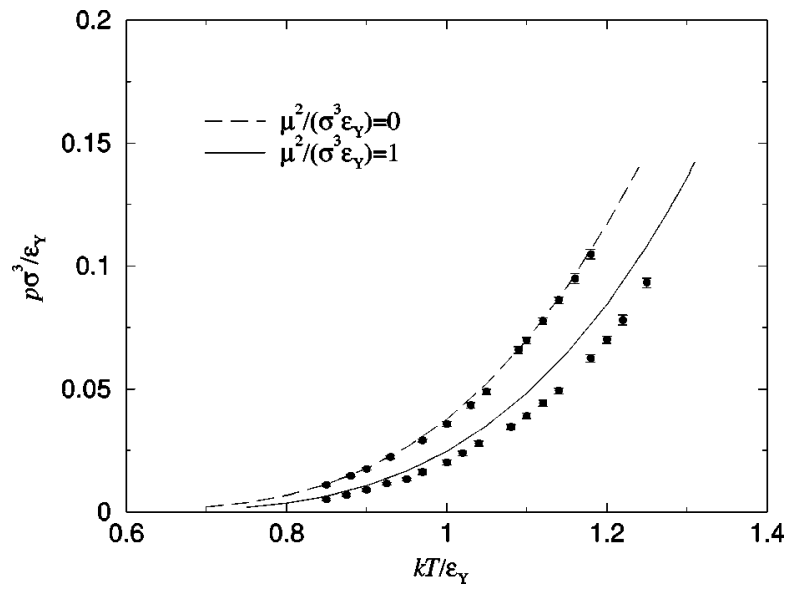

FIG. 5. Vapor pressure curves for the DYF for the two values of the dipole moment used in Fig. 4. The curves and points have the same meaning as in Fig. 4.

DHSF and therefore for the DYF too. It is more instructive to examine the extent to which $g_{S}(R)$ depends on $\mu$, and $h_{D}(R), h_{\Delta}(R)$ and $\epsilon$ depend on $\epsilon_{Y}$. Except near contact $(R=\sigma), g_{S}(R)$ is only weakly dependent on $\mu$. The angular functions, $h_{D}(R)$ and $h_{\Delta}(R)$, and $\epsilon$ are only weakly dependent on $\epsilon_{Y}$. This agrees with predictions of the MSA.

Hence, the errors in the MSA are most apparent in the values of $g_{S}\left(\sigma^{+}\right)$. If the MSA were exact, $g_{S}\left(\sigma^{+}\right)$would be independent of $\mu$. As seen in Table I, the change in $g_{S}\left(\sigma^{+}\right)$, with $\beta \epsilon_{Y}=1$, for $\beta \mu^{2} / \sigma^{3}$ changing from 0 to 2 is not greater than with $\beta \epsilon_{Y}=0$ for the same change in $\beta \mu^{2} / \sigma^{3}$. Thus, the MSA error for the DYF is not worse than that for the DHSF.

As opposed to the DHSF, the liquid-vapor equilibrium exists in the DYF. The MSA and simulation results for the coexistence curve has been compared. The MSA prediction agrees well with the simulation results.

The DYF seems as useful as the DHSF was once thought to be and the DYF is, under most circumstances, free of chain-like structures seen in the DHSF. Such structures are not present in the STMF if the dispersion force is strong enough. This is true for the DYF if $\epsilon_{Y}$ is large enough relative to $\mu$. Variation of $\lambda$ may also affect the existence of

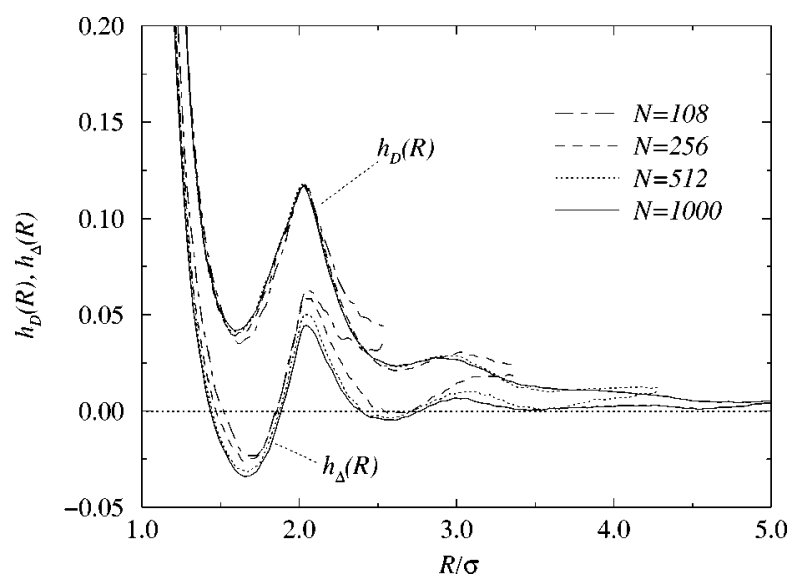

FIG. 6. $h_{D}(R)$ and $h_{\Delta}(R)$ of the DHSF $\left(\beta \epsilon_{Y}=0\right)$ at $\beta \mu^{2} / \sigma^{3}=1$ for various numbers of particles. 
chain-like structures in the DYF. This question and further work on the thermodynamic properties for the DYF are under study.

The fact that the properties of the DYF can be obtained from analytic formulas using the MSA is an important advantage of the DYF as compared to the similar STMF. For example, the DYF could be used as a reference fluid in a perturbation theory. To use the STMF in such an application requires the use of some numerical fit of the STMF properties that involves a large number of parameters. Furthermore, the range parameter of the YF can be varied rather easily. The counterpart of this in the STMF fluid is to change one or more of the indices 12 and 6 in the Lennard-Jones potential, the consequences of which are not well studied.

\section{ACKNOWLEDGMENTS}

The research was supported by the Research Grants Council of Hong Kong (HKU249/95P), by the National Science Foundation (Grant CHE96-01971), and by the donors of the Petroleum Research Fund, administered by the American Chemical Society (Grant No. ACS-PRF 31573-AC9). DH is a John Simon Guggenheim Memorial Foundation Fellow and is grateful for this support.
${ }^{1}$ M. S. Wertheim, J. Chem. Phys. 55, 4291 (1971).

${ }^{2}$ L. Onsager, J. Am. Chem. Soc. 58, 1486 (1936).

${ }^{3}$ L. Blum, J. Chem. Phys. 61, 2129 (1974).

${ }^{4}$ J. M. Caillol, J. Chem. Phys. 98, 9835 (1993); M. E. van Leeuwen and B. Smit, Phys. Rev. Lett. 71, 3991 (1993); J. J. Weis and D. Levesque, ibid. 71, 2729 (1993)

${ }^{5}$ G. Stell, J. C. Rasaiah, and N. Narang, Mol. Phys. 23, 393 (1972); A full set of references is given in Ref. 6.

${ }^{6}$ G. Kronome, J. Liszi, and I. Szalai, J. Chem. Soc., Faraday Trans. 93, 3053 (1997)

${ }^{7}$ D. Henderson and W. Schmickler, J. Chem. Soc., Faraday Trans. 92, 3839 (1996)

${ }^{8}$ D. Henderson, E. Waisman, J. L. Lebovitz, and L. Blum, Mol. Phys. 35, 241 (1978).

${ }^{9}$ E. Waisman, Mol. Phys. 25, 45 (1973).

${ }^{10}$ J. S. Hbye and G. Stell, Mol. Phys. 32, 195 (1976); M. Ginoza, ibid. 71, 145 (1990); D. Henderson, L. Blum, and J. P. Noworyta, J. Chem. Phys. 102, 4973 (1995).

${ }^{11}$ J. A. Barker and R. O. Watts, Chem. Phys. Lett. 26, 789 (1973).

${ }^{12}$ C. Rey, L. J. Gallego, L. E. González, and D. J. González, J. Chem. Phys. 97, 5121 (1992); C. Rey, L. J. Gallego, and L. E. González, ibid. 96, 6984 (1992).

${ }^{13}$ D. Levesque, G. N. Patey, and J. J. Weis, Mol. Phys. 34, 1077 (1977).

${ }^{14}$ G. N. Patey, D. Levesque, and J. J. Weis, Mol. Phys. 45, 733 (1982).

${ }^{15}$ E. Lomba, C. Martin, and M. Lombardero, Mol. Phys. 77, 1005 (1992).

${ }^{16}$ J. A. Barker and D. Henderson, Mol. Phys. 21, 187 (1971).

${ }^{17}$ D. Boda, J. Liszi, and I. Szalai, Chem. Phys. Lett. 235, 140 (1995).

${ }^{18}$ D. Boda, J. Liszi, and I. Szalai, Mol. Phys. 85, 429 (1995).

${ }^{19}$ B. Smit and D. Frenkel, Mol. Phys. 74, 35 (1991); E. Lomba and N. G. Almarza, J. Chem. Phys. 100, 8367 (1994). 